\section{FRAXAC2 instability}

Sir — Last year in Nature Genetics, Zhong et al. ${ }^{1}$ described the molecular basis for polymorphism at FRAXAC2, a marker first characterized and then used in our laboratories for linkage disequilibrium studies in fragile $\mathrm{X}$ syndrome. We agree that this polymorphism is due to single base differences in length due to a combination of three polymorphic repeat sequences ${ }^{1}$ as distinct from our assumption that the repeat would be a two base pair (bp) polymorphism based on the $(\mathrm{AC})_{\mathrm{n}}$ repeat used to isolate this marker ${ }^{2}$.

However, Zhong et al. also claim a mutation rate in fragile $\mathrm{X}$ families of 3.3\% based on inconsistent inheritance of FRAXAC2 PCR products in two pedigrees. They state that "The difference in mutation frequency observed between fragile $\mathrm{X}$ female carriers $(4 / 121)$ and all others as controls $(0 / 160)$ was significant $\left(\chi^{2}=\right.$ 18.92 , d.f. $=1, \mathrm{p}<0.001)$ ". When we calculate the statistics on these mutation frequencies we find a corrected $\chi^{2},=$ 3.19 ( $p>0.07$ ), suggesting that the difference between their observed mutationfrequencies in fragileXfemale carriers versus controls is not significant. Furthermore, when comparing frequencies of relatively rare events, the volatility of such statistics is so high that any interpretation (or conclusion) can only be tentative at best.

We have analysed 132 (in Australia) and 179 (in France) female transmissions of the fragile $\mathrm{X}$ chromosome and have not observed a single change in FRAXAC2 band length. The odds against this observation (if the mutation frequency ${ }^{1}$ of $3.3 \%$ is correct) is $1.3 \times$ $10^{-6}$. If our numbers are added to those of Zhong et al. (which may not be appropriate -3 of their 4 mutations were observed in the offspring of a single carrier female who may be otherwise predisposed to such mutation) then the observed mutation frequency for FRAXAC2 in fragile $\mathrm{X}$ female carriers is $<1 \%$ (and considerably less if normal $\mathrm{X}$ chromosomes are added). This mutation frequency is consistent with observed mutation frequencies at other microsatellite loci $i^{3-5}$.

Zhong et al. found no evidence of linkage disequilibrium with
FRAXAC2 which is at odds with studies on a variety of fragile $X$ cohorts (of European descent) including some from New York (the location of the Zhong et al. study ${ }^{6-8}$ and contradict the strong linkage disequilibrium observed between FRAXAC1 and FRAXAC2 in both the different fragile $\mathrm{X}$ cohorts and populations of normal $\mathrm{X}$ chromosomes ${ }^{6}$.

In the family exhibiting 3 of the 4 observed FRAXAC2 mutations (one of which is not even on the fragile $X$ chromosome) another extremely rare event appears to have occurred: a reversion from a premutation to a completely normal allele. The clustering of these observations in the one family suggests that their molecular basis is unlikely to be of general significance to other fragile $\mathrm{X}$ families.

In conclusion, we do not believe that FRAXAC2 warrants the term 'highly mutable'. It may well be that this locus is a complex polymorphism, however we do not believe that its properties constitute part of the fragile $\mathrm{X}$ mutation process, at least as outlined in Zhong et al.

\section{R.I. Richards}

K. Holman

K. Friend

\section{A. Staples}

\section{G.R. Sutherland}

Centre for Medical Genetics,

Women's and Children's Hospital,

72 King William Road,

North Adelaide,

Southern Australia 5006, Australia

\section{Oudet}

\section{Biancalana}

J.-L. Mandel

Inserm Unité 184, and LGME/

CNRS,

Faculte de Medecine,

Université Louis Pasteur,

11 Rue Humain,

67085 Strasbourg, France

Sir - The fragile $\mathrm{X}$ locus locus is flanked by two CA repeat-loci, FRAXAC1 and FRAXAC2, 10 kilobases from the expanded (CGG) repeat, on the centromeric and telomeric sides, respectively ${ }^{2}$. On the basis of polymorphisms exhibited by these CA repeats, we and others $\mathrm{s}^{6,8,9}$ have shown that some founder chromosomes are at the origin of
fragileX syndrome in the population. Zhong et al. ${ }^{1}$ recently showed that the FRAXAC2 locus was a hypermutable sequence composed of three variable subregions $(\mathrm{GT})_{\mathrm{x}},(\mathrm{TA})_{\mathrm{y}}$ and $(\mathrm{T})_{\mathrm{z}}$. Inheritance studies showed that this microsatellite seemed stable in normal families but unstable in female fragile $\mathrm{X}$ derived meioses, suggesting that the use of FRAXAC2 in linkage disequilibrium studies is not valid.

We have analysed 140 female fragile $\mathrm{X}$ derived meioses, 96 on the fragile $\mathrm{X}$ chromosome and the others on the normal X chromosome. We found no evidence of de novo mutations, suggesting that FRAXAC2 alleles are also stable in fragile $\mathrm{X}$ families. Comparison of our data with those of Zhong et al. did not reveal significant differences $\quad\left(\chi^{2}=2.25 \quad\right.$ [Yate's correction], d.f. $=1 ; p>0.1)$. Our reanalysis of their data showed that the difference in mutation frequency observed between fragile $\mathrm{X}$ female carriers $(4 / 121)$ and all others as controls $(0 / 184)$ was poorly significant $\left(\chi^{2}=3.87\right.$, d.f. $=1 ; \mathrm{p}=0.05$ $($ not $\mathrm{p}<0.001)$ ). Thus, more data should be analysed before a final conclusion can be drawn about the instability of this locus. The fact that three of the four de novo mutations described by Zhong et al. were found in the same family suggests that the observed instability was likely a familial aberrant case. The last case, observed in another family, may be easily explained by a recombination between maternal $\mathrm{X}$ chromosomes.

Zhong $e$ t al. suggested that the rate of de novo mutations on the FRAXAC2 locus is around $3 \%$ in fragile $\mathrm{X}$ families. Such a high mutation rate would completely blur, in a few generations, allelic associations created on fragile $X$ founder chromosomes. Analysis of 272 fragile $\mathrm{X}$ chromosomes at FRAXAC2showed strong associations between FRAXAC2, FRAXA (CGG) and FRAXAC1 or DXS548, another CA repeat localized $150 \mathrm{~kb}$ centromeric of CGG. It is clear that such associations would not continue for more than a few generations if the mutation rate at FRAXAC2 is around $3 \%$. It is impossible that random mutations at the $(\mathrm{GT})_{\mathrm{x}},(\mathrm{TA})_{\mathrm{y}}$, and (T) sequences lead, by chance, to the same FRAXAC2 allele on fragile $\mathrm{X}$ chromosomes. To explain that denovo mutations occurred only on fragile $\mathrm{X}$ chromosomes, Zhong et al. suggest that FMR-1 may play a role in 